\title{
3rd ICTs and Society Meeting; Paper Session - Theorizing the Internet; Paper 1: Toward Trust as Result. A Transdis- ciplinary Research Agenda for the 'Future Internet'
}

\section{${ }^{1}$ Stefano De Paoli, ${ }^{2}$ G. R. Gangadharan, ${ }^{1}$ Aphra Kerr, ${ }^{3}$ Vincenzo D'Andrea, ${ }^{4}$ Martin Serrano, ${ }^{4}$ Dmitri Botvich, ${ }^{4}$ Jimmy McGibney}

${ }^{1}$ National University of Ireland, Maynooth, Sociology Department, Maynooth, Ireland

\{stefano.depaoli, aphra.kerr\}@nuim.ie

${ }^{2}$ Politecnico di Milano, Department of Electronics and Informatics, Milan, Italy

gangadharan@elet.polimi.it

${ }^{3}$ University of Trento, Department of Engineering and Computer Science and Department of Sociology, Trento, Italy vincenzo.dandrea@unitn.it

${ }^{4}$ Waterford Institute of Technology, Telecommunications Software and Systems Group. ArcLabs Ireland, West Campus, Waterford, Ireland

\{jmserrano,dbotvich\}@tssg.org

\begin{abstract}
Trust has emerged as one of the key challenges for the Future Internet and as a key theme of European research. We are convinced that a transdisciplinary research agenda - that we define to as Trust as Result - shared by Sociology and Computer Science, is of paramount importance for devising sustainable Trust solutions for the (Future) Internet stakeholders. The scope of this paper is to present some elements we consider important for building such an agenda.
\end{abstract}

Keywords: Trust, Future Internet, Trust Modelling, Trust as Result, Assemblage

Acknowledgement: The work by Stefano De Paoli, Aphra Kerr, Martin Serrano and Dmitri Botvich has received support from the Higher Education Authority (HEA) in Ireland under the PRTLI Cycle 4 programme, in the FutureComm project (Serving Society: Management of Future Communications Networks and Services).

The Future Internet is today one of the main research and industry challenges in Europe and World Wide (http://www.futureinternet.eu/). In particular the focus of Future Internet research is to redesign several aspects of the original Internet in order to support the future use and continuous growth of the Internet and the increased and increasing number of users world-wide. Approaching the challenges of the Future Internet requires a common effort of different disciplines and actors, as the Future Internet does not just involve technological problems but has implica- tions for the economy, society and governance.

Trust is one of the key challenges for the Future Internet as research focuses on the development of resilient and trustworthy Future Internet able to support Trust decision for users (European Commission, 2008). We are convinced that a transdisciplinary research agenda - that we define to as Trust as Result shared by Sociology and Computer Science, is of paramount importance for devising sustainable Trust solutions for the (Future) Internet stakeholders. 


\section{Scope of the Paper}

This paper outlines what we consider the main aspects for building a Trust transdisciplinary agenda. In particular, we distinguish our agenda by comparison with one of the current mainstream interdisciplinary approaches to Trust, that we define to as 'Trust Modelling'. Trust Modelling approaches assume Trust to be the input of the design of trustworthy ICTs, focusing therefore on Trust as a decontextualized 'object' (with no relation whatsoever with situation and context). We propose a different point of view that focuses not on taking Trust as input, but on how we can obtain Trust as the result (output) of the design.

Our goal is to conceive, develop and implement an innovative, transdiciplinary and visionary agenda for the "design-for-socialtrust" technology whose goal is to enable a novel, collaborative, and socially accepted way of creating Trust for the Future Internet. Our vision consider not bridging disciplinary differences into an interdisciplinary approach (like Trust modeling that takes some elements from social sciences and some from computing), but rather building from the beginning a shared repertoire of concepts and actions.

We believe that a good approach to conceptualizing Trust would in-fact be that of seeing it as an effect (a result) of the interrelations between social and technical elements and not as a sort of objective (pre)condition for interaction. Therefore, Trust has to be built together with the interrelation of various sociotechnical entities and not as its presupposition (as for Trust Modelling). Trust is not an essence, which explains social order (explanans) and that can be captured and objectfied, but rather an outcome (explanandum) the result - of the relations among entities. This perspective can lead to the design of novel systems that supports understanding of Trust (and also mistrust) in practice in different Internet domains.

\section{The Assemblage Theory}

We begin by conceptualizing Trust as a collective socio-technical product (which is based both and at once on an array of humans and non-humans entities). For addressing this human/non-human symmetry we need con- cepts able to cut across the common assumed distinction between technology and society. For our specific work we rely on the concept of Assemblage as developed in the work by Manuel DeLanda, (2002 and 2006).

The concept of assemblage considers that social and technological entities should be characterized not on the basis of their essential properties and necessary relations as happens with for example the concept of 'system'. The dynamics of an assemblage are different from that of a system as the entities composing the assemblage are characterized on the basis of what they are capable of doing when they interact with one another. These capacities depend on the entity properties but cannot be reduced to them since they involve an interrelation with other interacting entities. The concept of assemblage is in particular useful to investigate the processes and interrelational dynamics encompassing changes according to the different roles of entities in different assemblages. In other words, what we have is a flat ontology - as opposed to a hierarchical ontology of systems - in which the relations of an assemblage can change at any time and the parts can withdraw from one assemblage and enter into other assemblages even with different roles.

\section{Trust as Result Agenda}

We summarize now the main aspects of the research agenda proposed here. Our intention is to further develop these aspects in future writings and collaborations in concrete projects. For us moving toward a transdisciplinary agenda for Trust for the Future Internet requires re-articulate and re-conceptualize the following elements of current design of Trust:

I. It is important to focus on Trust as the outcome of the design (Trust as Result), and not on Trust as a precondition (as input) embedded in a model, subsequently implemented in computer systems. This Trust as Result can be achieved not just by integrating different disciplines, but rather with a movement that transcends disciplinary boundaries.

II. It is important for disciplines to work and collaborate on the creation and en- 
hancement of a transdisciplinary conceptual framework for Trust as Result grounded on a shared repertoire of concepts. This can be achieved by identifying since the beginning concepts that can serve this scope.

To achieve the previous goals:

III. it is important to focus on the hybrid nature of Trust, that involves already a mixture of technical and social, and therefore to reject a separation between a technical and a social object and the disciplinary differences implied by the existence of different objects of study.

IV. Finally it is important to rely on concepts that transcend common assumed separations. In our case we argue for a flat ontology based on the Assemblage Theory for designing Trust.

In conclusion, we are aware that arguing for a possible paradigm shift - as proposed in our agenda - in the design of Trustworthy ICT for the Future Internet requires more than just reshaping a few assumptions of the design, leaving intact the current core elements of systems design theories. Shifting from Trust Modelling (Trust as Input) to Transdisciplinary Trust (Trust as result) implies also a politics of transdisciplinarity. Indeed, we live in a world populated by Epistemic Cultures (KnorrCetina, 1999), in which how we know and what we know is often difficult to change. Current thinking in term of Trust as it relates to ICTs is strongly bounded with a core set of assumptions that cannot just be modified on the basis of pure technology success. Therefore enhancing a transdiciplinary Trust agenda for the Future Internet is also a political problem. We do not have yet an answer on how to solve the techno-political challenges we are facing, but we are convinced of the importance of working toward this goal and this paper is a first concrete effort in this direction.

\section{References}

DeLanda, M. (2002). Intensive Science and Virtual Philosophy. London: Continuum.

DeLanda, M. (2006). A New Philosophy of Society: Assemblage Theory and Social Complexity. London: Continuum.

European Commission (2008). Future Internet Compendium of Research Projects. Retrieved from: $\mathrm{ftp}: / / f t p . c o r d i s . e u r o p a . e u / p u b / f p 7 / i c t / d o c s / c h 1-g 848-280-f u t u r e-i n t e r n e t \_e n . p d f$

Knorr-Cetina, K. (1999). Epistemic Cultures: How the Sciences Make Knowledge. Cambridge: Harvard University Press.

\section{About the Authors}

Stefano De Paoli: http://www.nuim.ie/nirsa/people/postdocs/stefano de paoli.shtml

G. R. Gangadharan: http://sites.google.com/site/geeyaar2/

Aphra Kerr: http://sociology.nuim.ie/AphraKerr.shtml

Vincenzo D’Andrea: http://disi.unitn.it/users/vincenzo.dandrea

Martin Serrano: http://www.tssg.org/people/jmserrano/

Dmitri Botvich: http://www.tssg.org/people/dbotvich/

Jimmy McGibney: http://www.tssg.org/people/jmcgibney/ 